\title{
Intrinsic brainstem schwannoma - A rare clinical entity and a histological enigma
}

\author{
Anil Kumar Sharma, Amey R. Savardekar, B. N. Nandeesh'1, A. Arivazhagan, Malla Bhaskar Rao \\ Departments of Neurosurgery and ${ }^{1}$ Neuropathology, National Institute of Mental Health and Neurosciences, Bengaluru, Karnataka, India
}

\section{ABSTRACT}

Intraparenchymal schwannomas arising in the brainstem are very rare, and only eight cases have been reported in literature till now. We report an intraparenchymal brainstem schwannoma presenting with the classical clinical presentation of an intrinsic brainstem lesion, and discuss its clinicoradiological characteristics and histological origins. We highlight the importance of an intraoperative frozen section diagnosis in such cases. Intraoperative tissue diagnosis significantly may alter the surgical strategy, which should be aimed at near total intracapsular decompression of the schwannoma.

Key words: Intraparenchymal schwannoma, intrinsic brainstem schwannoma, pathogenesis of schwannoma

\section{Introduction}

Schwannomas arise from the myelin sheath of peripheral nerves and account for $8-10 \%$ of all intracranial tumors. ${ }^{[1]}$ Most commonly encountered in extra-axial locations, intra-axial schwannomas are rare and arouse significant histological curiosity. ${ }^{[1,2]}$ They account for only $1-2 \%$ of intracranial schwannomas and have been reported in periventricular and cerebellum but very rarely as intrinsic brainstem lesions..$^{[1,3,4]}$ We report an unusual schwannoma, presenting as a classical intrinsic brainstem lesion, and discuss its clinicoradiological characteristics and histological origins.

\section{Case Report}

A 26-year-old female presented with headache, progressive gait ataxia, right hemiparesis, left facial paresis, and slurring of speech of 7 months duration. The neurological examination showed papilledema,

\section{Address for correspondence:}

Dr. Amey R. Savardekar, Department of Neurosurgery, National Institute of Mental Health and Neurosciences, Bengaluru, Karnataka, India. E-mail: ameysavardekar@gmail.com

\begin{tabular}{|l|l|}
\hline \multicolumn{2}{|c|}{ Access this article online } \\
\hline Quick Response Code: & Website: \\
\hline & www.ruralneuropractice.com \\
\cline { 2 - 2 } & \\
\hline
\end{tabular}

coarse rotatory nystagmus with fast component to right, left paramedian pontine reticular formation nucleus involvement with left lateral gaze palsy, left facial weakness (House and Brackmann Grade 4), asymmetrical palatal weakness (right more than left), impaired gag reflex, bilateral appendicular cerebellar signs, broad-based gait, truncal ataxia, and right hemiparesis (4/5 weaknesses on the 5-point strength scale). Clinical impression was of intrinsic brainstem pathology.

Magnetic resonance imaging (MRI) of the brain showed an intra-axial, solid-cystic lesion involving the pons and medulla, extending up to cervicomedullary junction. The solid portion was hypointense on T1-weighted images, hyperintense on T2-weighted images and showed enhancement on contrast administration. Cystic components were cerebrospinal fluid isointense. The fourth ventricle was compressed and displaced posteriorly by the lesion [Figure 1a-d].

The patient underwent midline suboccipital craniotomy in prone position, with the intent of decompressing the lesion and achieving a histopathological diagnosis. After dural opening and telovelotonsillar approach, the

This is an open access article distributed under the terms of the Creative Commons Attribution-NonCommercial-ShareAlike 3.0 License, which allows others to remix, tweak, and build upon the work non-commercially, as long as the author is credited and the new creations are licensed under the identical terms.

For reprints contact: reprints@medknow.com

How to cite this article: Sharma AK, Savardekar AR, Nandeesh BN, Arivazhagan A, Rao MB. Intrinsic brainstem schwannoma - A rare clinical entity and a histological enigma. J Neurosci Rural Pract 2016;7:302-4. 


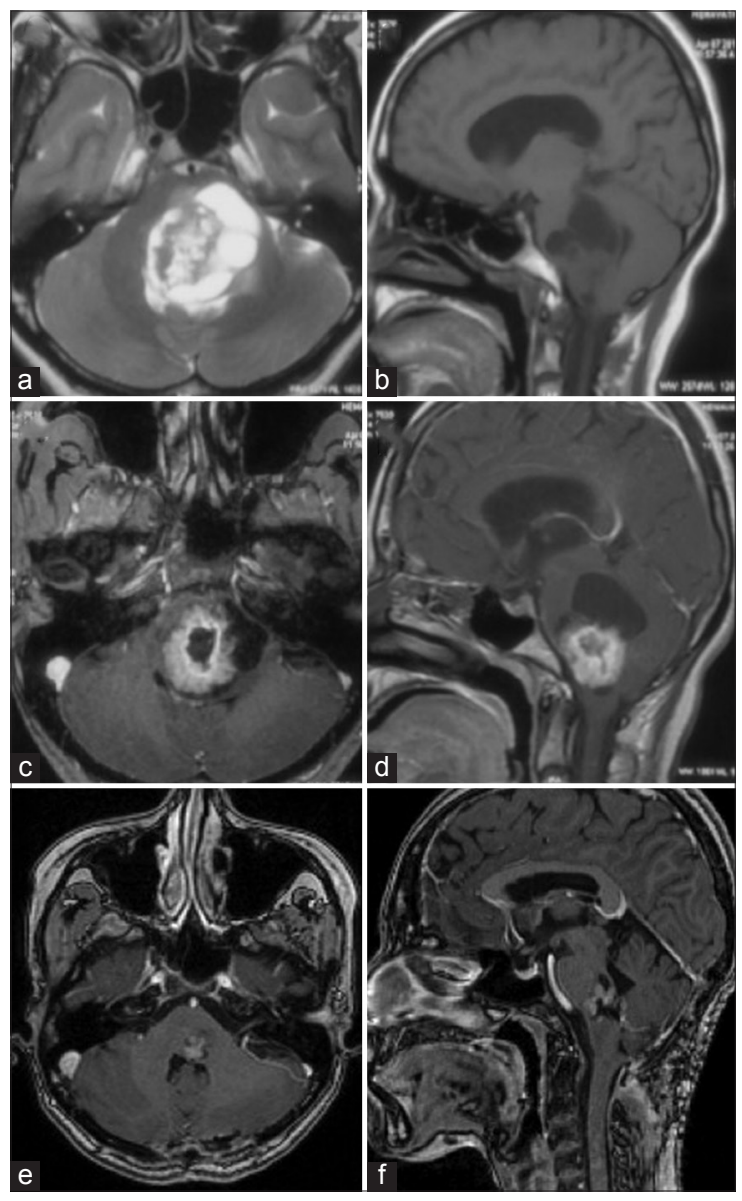

Figure 1: (a-d) Preoperative magnetic resonance imaging, (a) T2-weighted axial, (b) precontrast T1-weighted sagittal, (c) postcontrast T1-weighted axial, (d) postcontrast T1-weighted sagittal, showing a well-demarcated, intra-axial, solid-cystic tumor involving pons and medulla. (e and f) Magnetic resonance imaging at 6 months follow-up, postcontrast T1-weighted axial (e), and sagittal (f) images, showing small enhancing residual tumor component at left pontomedullary junction

bulging floor of the fourth ventricle was encountered in the operative field. Incision was taken at the most translucent bulging part of the fourth ventricular floor, overlying the cystic portion of the tumor, after electrical stimulation for facial nerve function. Tumor was suckable, moderately vascular with solid cystic components. After the intraoperative frozen section examination had revealed schwannoma, attempt was made for maximal safe resection. Patient's postoperative course was uneventful, without any fresh neurological deficits.

Histopathology [Figure 2a] revealed spindle cell neoplasm composed of lobules of interlacing fascicles of spindle cells having a twisted buckled nuclei and fibrillary cytoplasm morphologically resembling Schwann cells. On immunohistochemistry, the tumor cells exhibited intense S-100 positivity, and this

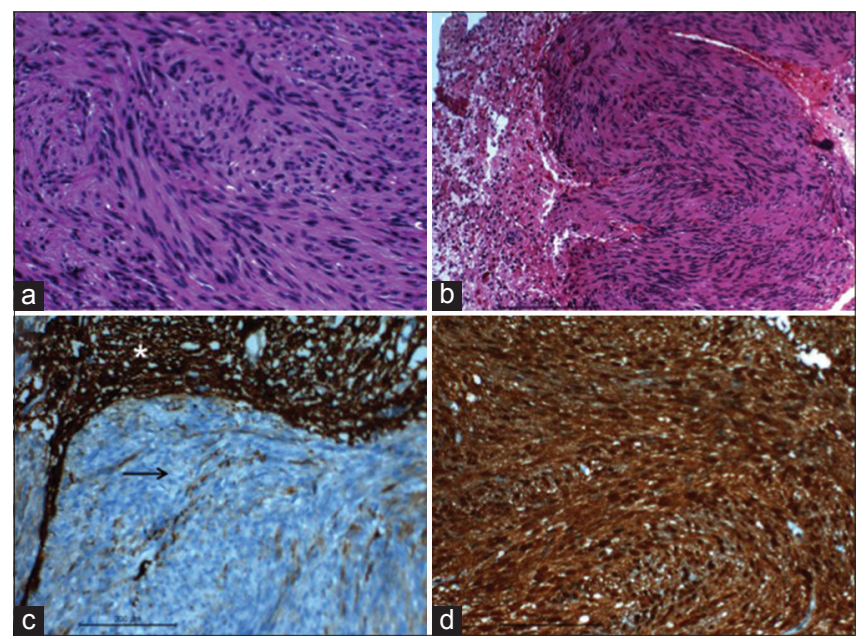

Figure 2: (a) Microphotograph showing the twisted/buckled nuclear characters of the spindle cells and the fibrillated cytoplasm $(\mathrm{H}$ and $\mathrm{E}$, $\times 400$ ); (b) microphotographs showing the neoplasm focally infiltrating the glial parenchyma ( $\mathrm{H}$ and $\mathrm{E}, \times 200$ ); (c) microphotograph showing the tumors cells not stained with glial fibrillary acidic protein (black arrow), however the stain highlights the adjacent glial tissue (asterisk) (IHC stain glial fibrillary acidic protein, $\times 200$ ); (d) microphotograph showing the tumor cells stained with S100 (IHC S100, ×200)

confirmed the diagnosis of schwannoma [Figure 2d]. The lobules were reticulin-rich. Focal brain invasion was noted which showed edema and reactive gliosis [Figure 2b].

At 6 months follow-up, the patient has improved symptomatically with residual left lateral gaze paresis and left partial facial nerve palsy. MRI at 6 months follow-up [Figure 1e and f] shows a small residual lesion, without any mass effect. She is advised regular follow-up with periodic MRI of the brain.

\section{Discussion}

There are eight reported cases of intrinsic brainstem schwannomas, in English literature to the best of our knowledge, making this an extremely rare entity ${ }^{[4,5]}$ Intraparenchymal schwannomas are extremely uncommon lesions and in contrast to vestibular schwannomas, tend to occur in young adults during the second and third decades ${ }^{[4]}$ The clinical manifestations of intraparenchymal schwannoma depend mainly on the location and size of the tumor. They are well-demarcated lesions with approximately $15 \%$ cases being associated with a significant cystic component. ${ }^{[2]}$

MRI characteristics may be variable, but usually intrinsic schwannomas are radiologically similar to extra-axial schwannomas; being hypo- or iso-intense on T1-weighted images, hyperintense on T2-weighted images and the 
solid portion demonstrating intense enhancement after contrast administration. ${ }^{[5]}$ There are no pathognomic neuroimaging features of intra-axial schwannomas, and hence, these cases are almost always preoperatively diagnosed as one of the more common differentials.

In our case, the preoperative diagnosis was an intrinsic brainstem glioma (focal or dorsally exophytic glioma, probably low grade). The importance of getting a frozen section diagnosis intraoperatively in such cases cannot be over-emphasized, since the diagnosis of a benign entity-like schwannoma, may alter the surgical strategy. Histologically, intra-axial schwannomas are analogous to extra-axial (commonly vestibular) schwannomas; however, immunohistochemistry usually plays an important role in clinching the diagnosis, as they exhibit intense immunoreactivity to S-100 [Figure 2d] and vimentin, and no reactivity to glial fibrillary acidic protein [Figure $2 \mathrm{c}] \cdot{ }^{[4]}$ The invasion of the normal brain parenchyma seen in our case [Figure $2 b$ ], cautions against any attempt for total resection in such cases. Intracapsular decompression and leaving a thin sliver of tumor tissue peripherally may be a good surgical strategy to achieve a satisfactory postoperative outcome.

The histogenesis of an intrinsic brainstem schwannoma continues to be an enigma as Schwann cells are not present within the brain parenchyma. ${ }^{[3,4]}$ The theories proposed to explain their origin can be classified as developmental and nondevelopmental. According to the developmental theory, mesenchymal (pial) cells or ectopic neural crest cells in the brain parenchyma can transform into foci of Schwann cells and serve as a source of origin for intraparenchymal schwannoma. Another theory is the occurrence of a developmental abnormality during closure of the neural tube in the $4^{\text {th }}$ gestational week, resulting in hamartomatous foci of Schwann cells within the brain parenchyma, from which intrinsic schwannomas may arise. Nondevelopmental theories are based on the fact that Schwann cells are present within the perivascular autonomic nervous plexus and large arteries in the subarachnoid spaces and may give rise to schwannomas. Similar tiny myelinated nerve plexi are common in tela choroidea, which may help to explain the predilection of intraparenchymal schwannomas for periventricular sites. ${ }^{[3]}$ Some authors have suggested the existence of aberrant intramedullary peripheral nerve fibers that may lead to proliferation of Schwann cells in the presence of chronic stimulus, such as inflammation, leading to an intrinsic schwannoma. ${ }^{[4]}$ Yet another theory suggests that the origin of intrinsic schwannoma could be from Schwann cells in the root entry zone of cranial nerves, which may, albeit rarely, grow preferentially into the subpial space of the brainstem. ${ }^{[5]}$

\section{Conclusion}

Intrinsic brainstem schwannomas are exceedingly rare. Preoperative diagnosis remains elusive as there are no clinical or radiological pathognomic features which may differentiate it from an intrinsic, low-grade glioma (focal or dorsally exophytic). Intraoperative frozen section is essential in establishing an intraoperative diagnosis and may significantly alter the surgical strategy, which should ideally be near total intracapsular decompression of the schwannoma.

\section{Financial support and sponsorship}

Nil.

\section{Conflicts of interest}

There are no conflicts of interest.

\section{References}

1. Lin J, Feng H, Li F, Zhao B, Guo Q. Intraparenchymal schwannoma of the medulla oblongata. Case report. J Neurosurg 2003;98:621-4.

2. Muzzafar S, Ketonen L, Weinberg JS, Schellingerhout D. Imaging and clinical features of an intra-axial brain stem schwannoma. AJNR Am J Neuroradiol 2010;31:567-9.

3. Paredes I, Jimenez Roldán L, Ramos A, Lobato RD, Ricoy JR. Intraparenchymal schwannomas: Report of two new cases studied with MRI and review of the literature. Clin Neurol Neurosurg 2012;114:42-6.

4. Ramos AA, Vega MA, Valencia HS, García JC, Perez VC. Intraparenchymal schwannoma involving the brainstem in a young woman. Pediatr Neurol 2013;48:472-4.

5. Srivastav AK, Tungaria A, Kumar R, Sahu RN. Intraparenchymal schwannoma of brainstem in a pediatric patient. Neurol India 2011;59:924-6. 